\title{
Abstracts from the Clinical Audit and Practice Advisory Group Annual Audit and Evidence-Based Medicine Meeting, at the ENT UK Meeting, 9 September 2010, University Hospital Coventry, UK
}

\author{
ENT surgical patients benefit from an anaesthetist-led \\ pre-admission clinic: a prospective, closed-loop audit \\ over two years \\ D Owens, S Le Maitre, S S Backhouse \\ From the Princess of Wales Hospital, Bridgend
}

\section{Background}

In the Princess of Wales Hospital, most ENT patients are admitted on the day of surgery, following a preadmission clinic assessment by an ENT junior doctor. We found that, even with this assessment, many patients had their operation postponed on the day of surgery as they were medically unfit. Subsequently, a hospital-wide, anaesthetist-led pre-admission clinic was established for all surgical patients.

This audit examined the incidence of patient cancellations on the day of surgery, and the impact of our new anaesthetist-led pre-admission clinic on this incidence.

\section{Method}

A prospective, closed-loop audit of ENT admissions was undertaken. We collected one year of data for each type of pre-admission clinic: the original preadmission clinic (data from May 2007 to April 2008) and the anaesthetist-led pre-admission clinic (data from May 2008 to April 2009). For each data collection period, we documented day-of-surgery patient cancellations and the reasons for them. Results were statistically analysed using the chi-square evaluation.

Results

A total of 500 patients were recruited: 290 during the first data collection period and 210 during the second. Day-of-surgery patient cancellations dropped from 11.7 per cent (confidence interval (CI) 4.5-15.7) to 8.1 per cent (CI 6.8-19.5) over the audit period. Day-of-surgery cancellations due to the anaesthetist deciding that the patient was unfit for surgery dropped from 3.79 per cent (CI 2.1-6.7) to 0.5 per cent (CI 0.01-2.6). Chi-square evaluation showed that this improvement in cancellation rates was statistically significant $(\mathrm{chi}=5.721, p=0.017)$.

\section{Conclusion}

This audit loop showed that the incidence of day-ofsurgery patient cancellations reduced when pre-admission assessment was undertaken by anaesthetists. This had substantial benefits for the patients and the hospital. We recommend the introduction of anaesthetistled pre-admission clinics to reduce day-of-surgery patient cancellations

\begin{abstract}
Does an aide memoire improve recording of consent by senior house officers?
\end{abstract}

R J List, D Siau, M Motammed

From Fairfield General Hospital, Bury, UK

\section{Objectives}

To audit the risks recorded on otorhinolaryngology consent forms, and to assess the impact of an aide memoire provided to senior house officers (SHOs).

Methods

We conducted a prospective audit cycle of 94 consent forms for patients listed for myringoplasty, endoscopic sinus surgery, septoplasty and oesophagoscopy. The risks stated in ENT UK patient information leaflets were used as audit standards.

\section{Result}

Prior to provision of the aide memoire, SHOs recorded 48 per cent of the standard risks, while non-SHO grades recorded 49 per cent. Following provision of the aide memoire, SHO risk recording rose to 84 per cent, compared with 58 per cent in the non-SHO group.

\section{Conclusion}

Provision of an aide memoire to SHOs can result in significant improvement in the number of standard risks they record on otorhinolaryngology consent forms. Such a resource can be easily provided at the time of departmental induction. 